\title{
Partitioning Technique for Relaxed Stability Criteria of Discrete-Time Systems with Interval Time-Varying Delay
}

\author{
Kuang-Yow Lian, ${ }^{1}$ Wen-Tsung Yang, ${ }^{2}$ and Peter Liu ${ }^{3}$ \\ ${ }^{1}$ Department of Electrical Engineering, National Taipei University of Technology, Taipei 10608, Taiwan \\ ${ }^{2}$ Department of Electrical Engineering, Chung-Yuan Christian University, Chung-Li 32023, Taiwan \\ ${ }^{3}$ Department of Electrical Engineering, Tamkang University, New Taipei City 25137, Taiwan
}

Correspondence should be addressed to Kuang-Yow Lian; kylianntut@gmail.com

Received 23 March 2014; Accepted 10 June 2014; Published 30 June 2014

Academic Editor: Mustafa Kulenović

Copyright (C) 2014 Kuang-Yow Lian et al. This is an open access article distributed under the Creative Commons Attribution License, which permits unrestricted use, distribution, and reproduction in any medium, provided the original work is properly cited.

\begin{abstract}
We demonstrate an improved stability analysis based on a partition oriented technique for discrete-time systems with interval time-varying delay. The partition oriented technique introduces beneficial terms contributing to the negative definiteness of the Lyapunov function difference, meanwhile completely avoiding traditional inequality based approaches. In contrast, nonpartitioning oriented techniques do not put emphasis on further dividing the interval of the summation in the Lyapunov function. Herein, we demonstrate that the advantages of exploiting partitioning techniques manifest the relaxed stability criteria, as well as the flexibility to tune tradeoff between allowable timedelay range performance and computational load. Simulation carried out on a benchmark discrete-time system reveals the significant improvement in terms of maximum allowable upper bound in comparison.
\end{abstract}

\section{Introduction}

Stability analysis on continuous and discrete-time systems with interval time-varying delay has been extensively researched in the past decade [1-7]. Traditional techniques focused on introducing inequalities (e.g., Moon et al. [8], Park [9], model transformation $[10,11])$ to cope with the double integral problem in the Lyapunov function whereas we term these approaches as nonpartitioning oriented techniques. However, these nonpartitioning oriented techniques increase conservativeness by introducing positive terms to the Lyapunov function difference. This setback led to techniques (e.g., zero equalities [12], averaging technique [13]) which further partition the time-delay interval to introduce beneficial terms contributing to the negative definiteness of the Lyapunov function difference (whereas we term this as partitioning techniques), resulting in relaxed stability criteria. From the above observation, we are motivated to completely avoid nonpartitioning oriented techniques while exploiting partitioning oriented techniques. The advantages are (i) relaxed stability criteria and (ii) flexibility to tune tradeoff between allowable time-delay range performance and computational load. Simulation results on a discretetime example reveal the best performance in comparison to previous literature.

\section{Main Results}

Using the partitioning technique, we have the following main results.

Theorem 1. Given nonnegative integer scalars $\tau_{M}$ and $\tau_{m}$, the system

$$
\begin{gathered}
x(k+1)=A x(k)+A_{h} x(k-h(k)), \\
x(k)=\varphi(k), \quad k \in\left[-\tau_{M}, 0\right]
\end{gathered}
$$

is asymptotically stable, where $x(k) \in \mathbf{R}^{n}$ is the state vector; $A, A_{h}$ are constant matrices with appropriate dimensions; $\varphi(k)$ is the initial condition of $k \in\left[-\tau_{M}, 0\right]$; for any time varying delay $h(k)$ satisfying $0 \leq \tau_{m} \leq h(k) \leq \tau_{M}$, there exist real matrices $P=P^{T}>0, Q_{i}=Q_{i}^{T}>0(i=1,2,3,4), R=$ $R^{T}>0, Z=Z^{T}>0$, and any appropriately dimensioned 
matrices $N=\left[N_{1}^{T}, N_{2}^{T}\right]^{T}, M=\left[M_{1}^{T}, M_{2}^{T}\right]^{T}, S=\left[S_{1}^{T}, S_{2}^{T}\right]^{T}$, and $T=\left[T_{1}^{T}, T_{2}^{T}\right]^{T}$ such that the following two sets of LMIs hold.

Case I $\left(0 \leq \tau_{m} \leq h(k) \leq \tau_{\text {avg }}\right)$. Consider

$$
\begin{aligned}
& \Theta_{1}=\left[\begin{array}{ccc}
X_{11} & X_{12} & N_{1} \\
X_{12}^{T} & X_{22} & N_{2} \\
N_{1}^{T} & N_{2}^{T} & Z
\end{array}\right] \geq 0, \\
& \Theta_{2}=\left[\begin{array}{ccc}
X_{11}+Y_{11} & X_{12}+Y_{12} & M_{1} \\
X_{12}+Y_{12}^{T} & X_{22}+Y_{22} & M_{2} \\
M_{1}^{T} & M_{2}^{T} & R+Z
\end{array}\right] \geq 0, \\
& \Theta_{3}=\left[\begin{array}{ccc}
X_{11}+Y_{11} & X_{12}+Y_{12} & S_{1} \\
X_{12}^{T}+Y_{12}^{T} & X_{22}+Y_{22} & S_{2} \\
S_{1}^{T} & S_{2}^{T} & R+Z
\end{array}\right] \geq 0, \\
& \Theta_{4}=\left[\begin{array}{ccc}
X_{11} & X_{12} & T_{1} \\
X_{12}^{T} & X_{22} & T_{2} \\
T_{1}^{T} & T_{2}^{T} & Z
\end{array}\right] \geq 0,
\end{aligned}
$$

$\Phi$

$$
=\left[\begin{array}{ccccc}
\Phi_{11} & \Phi_{12} & -N_{1}+M_{1} & -T_{1} & -S_{1}+T_{1} \\
\Phi_{12}^{T} & \Phi_{22} & -N_{2}+M_{2} & -T_{2} & -S_{2}+T_{2} \\
-N_{1}^{T}+M_{1}^{T} & -N_{2}^{T}+M_{2}^{T} & -Q_{2} & 0 & 0 \\
-T_{1}^{T} & -T_{2}^{T} & 0 & -Q_{3} & 0 \\
-S_{1}^{T}+T_{1}^{T} & -S_{2}^{T}+T_{2}^{T} & 0 & 0 & -Q_{4}
\end{array}\right]
$$$$
<0 \text {, }
$$

where

$$
\begin{aligned}
\Phi_{11}= & -P+Q_{1}+Q_{2}+Q_{3}+Q_{4}+\left(\tau_{M}-\tau_{m}\right) Q_{1}+N_{1}+N_{1}^{T} \\
& +\tau_{M} X_{11}+\delta_{1} Y_{11}+A^{T} P A+(A-I)^{T} \Theta(A-I), \\
\Phi_{12}= & N_{2}^{T}-M_{1}+S_{1}+\tau_{M} X_{12}+\delta_{1} Y_{12}+A^{T} P A_{h} \\
& +(A-I)^{T} \Theta A_{h}, \\
\Phi_{22}= & -M_{2}-M_{2}^{T}+S_{2}+S_{2}^{T}-Q_{1}+\tau_{M} X_{22}+\delta_{1} Y_{22} \\
& +A_{h}^{T} P A_{h}+A_{h}^{T} \Theta A_{h}, \\
\Theta=\delta_{1} R & +\tau_{M} Z .
\end{aligned}
$$

Case II $\left(\tau_{\text {avg }} \leq h(k) \leq \tau_{M}\right)$. Consider

$$
\begin{gathered}
\bar{\Theta}_{1}=\left[\begin{array}{lll}
X_{11} & X_{12} & N_{1} \\
X_{12}^{T} & X_{22} & N_{2} \\
N_{1}^{T} & N_{2}^{T} & Z
\end{array}\right] \geq 0, \\
\bar{\Theta}_{2}=\left[\begin{array}{lll}
X_{11} & X_{12} & M_{1} \\
X_{12}^{T} & X_{22} & M_{2} \\
M_{1}^{T} & M_{2}^{T} & Z
\end{array}\right] \geq 0, \\
\bar{\Theta}_{3}=\left[\begin{array}{ccc}
X_{11}+Y_{11} & X_{12}+Y_{12} & S_{1} \\
X_{12}+Y_{12}^{T} & X_{22}+Y_{22} & S_{2} \\
S_{1}^{T} & S_{2}^{T} & R+Z
\end{array}\right] \geq 0, \\
\bar{\Theta}_{4}=\left[\begin{array}{ccc}
X_{11}+Y_{11} & X_{12}+Y_{12} & T_{1} \\
X_{12}^{T}+Y_{12}^{T} & X_{22}+Y_{22} & T_{2} \\
T_{1}^{T} & T_{2}^{T} & R+Z
\end{array}\right] \geq 0,
\end{gathered}
$$

$\bar{\Phi}$

$$
=\left[\begin{array}{ccccc}
\Phi_{11} & \bar{\Phi}_{12} & -N_{1}+M_{1} & -T_{1} & -M_{1}+S_{1} \\
\bar{\Phi}_{12}^{T} & \bar{\Phi}_{22} & -N_{2}+M_{2} & -T_{2} & -M_{2}+S_{2} \\
-N_{1}^{T}+M_{1}^{T} & -N_{2}^{T}+M_{2}^{T} & -Q_{2} & 0 & 0 \\
-T_{1}^{T} & -T_{2}^{T} & 0 & -Q_{3} & 0 \\
-M_{1}^{T}+S_{1}^{T} & -M_{2}^{T}+S_{2}^{T} & 0 & 0 & -Q_{4}
\end{array}\right]
$$

$<0$,

where

$$
\begin{aligned}
\bar{\Phi}_{12}= & N_{2}^{T}-S_{1}+T_{1}+\tau_{M} X_{12}+\delta_{2} Y_{12} \\
& +A^{T} P A_{h}+(A-I)^{T} \Theta A_{h}, \\
\bar{\Phi}_{22}= & -S_{2}-S_{2}^{T}+T_{2}+T_{2}^{T}-Q_{1}+\tau_{M} X_{22} \\
& +\delta_{2} Y_{22}+A_{h}^{T} P A_{h}+A_{h}^{T} \Theta A_{h} .
\end{aligned}
$$

Proof. First, denote $\tau_{\text {avg }}=\left[(1 / 2)\left(\tau_{M}+\tau_{m}\right)\right]$, that is, the floor function of $(1 / 2)\left(\tau_{M}+\tau_{m}\right), \delta_{1}=\tau_{\text {avg }}-\tau_{m}$, and $\delta_{2}=\tau_{M}-\tau_{\text {avg }}$. Let

$$
y(l)=x(l+1)-x(l) .
$$

Then, it is clear that

$$
y(k)=x(k+1)-x(k)=(A-I) x(k)+A_{h}(k-h(k)) .
$$


We divide the proof into two cases.

Case I. We choose the Lyapunov function candidate

$$
V(k)=\sum_{i=1}^{5} V_{i}(k)
$$

where

$$
\begin{aligned}
V_{1}(k)= & x^{T}(k) P x(k), \\
V_{2}(k)= & \sum_{i=k-h(k)}^{k-1} x^{T}(i) Q_{1} x(i), \\
V_{3}(k)= & \sum_{i=k-\tau_{m}}^{k-1} x^{T}(i) Q_{2} x(i)+\sum_{i=k-\tau_{M}}^{k-1} x^{T}(i) Q_{3} x(i) \\
V_{4}(k)= & \sum_{j=-\tau_{M}+1}^{-\tau_{m}} \sum_{i=k+j}^{k-1} x^{T}(i) Q_{1} x(i), \\
V_{5}(k)= & \sum_{j=-\tau_{\text {avg }}}^{k-1} \sum_{i=k+j}^{k-1} y^{T}(i) R y(i)+\sum_{j=-\tau_{M}} x(i),
\end{aligned}
$$

and $P, Q_{1}, Q_{2}, Q_{3}, Q_{4}, R$, and $Z$ are positive definite matrices to be determined. Define $\Delta V_{i}(k)=V_{i}(k+1)-V_{i}(k)$ which leads to

$$
\begin{aligned}
& \Delta V_{1}(k)=x^{T}(k) A^{T} P A x(k)+x^{T}(k) A^{T} P A_{h} x(k-h(k)) \\
& +x^{T}(k-h(k)) A_{h}^{T} P A x(k)+x^{T}(k-h(k)) \\
& \times A_{h}^{T} P A_{h} x(k-h(k))-x^{T}(k) P x(k), \\
& \Delta V_{2}(k)=\sum_{i=k+1-h(k+1)}^{k-1} x^{T}(i) Q_{1} x(i)+x^{T}(k) Q_{1} x(k) \\
& -\sum_{i=k+1-h(k)}^{k-1} x^{T}(i) Q_{1} x(i) \\
& -x^{T}(k-h(k)) Q_{1} x(k-h(k)) \\
& \leq \sum_{i=k+1-\tau_{m}}^{k-1} x^{T}(i) Q_{1} x(i)+\sum_{i=k+1-\tau_{M}}^{k-\tau_{m}} x^{T}(i) Q_{1} x(i) \\
& +x^{T}(k) Q_{1} x(k)-\sum_{i=k+1-\tau_{m}}^{k-1} x^{T}(i) Q_{1} x(i) \\
& -x^{T}(k-h(k)) Q_{1} x(k-h(k)) \\
& =\sum_{i=k+1-\tau_{M}}^{k-\tau_{m}} x^{T}(i) Q_{1} x(i)+x^{T}(k) Q_{1} x(k) \\
& -x^{T}(k-h(k)) Q_{1} x(k-h(k)),
\end{aligned}
$$

$$
\begin{aligned}
\Delta V_{3}(k)= & x^{T}(k)\left(Q_{2}+Q_{3}+Q_{4}\right) x(k)-x^{T}\left(k-\tau_{m}\right) \\
& \times Q_{2} x\left(k-\tau_{m}\right)-x^{T}\left(k-\tau_{M}\right) Q_{3} x\left(k-\tau_{M}\right) \\
& -x^{T}\left(k-\tau_{\mathrm{avg}}\right) Q_{4} x\left(k-\tau_{\mathrm{avg}}\right),
\end{aligned}
$$

$$
\begin{aligned}
\Delta V_{4}(k)= & \left(\tau_{M}-\tau_{m}\right) x^{T}(k) Q_{1} x(k)-\sum_{i=k+1-\tau_{M}}^{k-\tau_{m}} x^{T}(i) Q_{1} x(i), \\
\Delta V_{5}(k)= & \left(\tau_{\text {avg }}-\tau_{m}\right) y^{T}(k) R y(k)-\sum_{i=k-\tau_{\text {avg }}}^{k-\tau_{m}-1} y^{T}(i) R y(i) \\
& +\tau_{M} y^{T}(k) Z y(k)-\sum_{i=k-\tau_{M}}^{k-1} y^{T}(i) Z y(i)
\end{aligned}
$$

From (6), the following zero equations

$$
\begin{gathered}
2 \zeta_{1}^{T}(k) N\left(x(k)-x\left(k-\tau_{m}\right)-\sum_{i=k-\tau_{m}}^{k-1} y(i)\right)=0, \\
2 \zeta_{1}^{T}(k) M\left(x\left(k-\tau_{m}\right)-x(k-h(k))-\sum_{i=k-h(k)}^{k-\tau_{m}-1} y(i)\right)=0,
\end{gathered}
$$

$$
2 \zeta_{1}^{T}(k) S\left(x(k-h(k))-x\left(k-\tau_{\mathrm{avg}}\right)-\sum_{i=k-\tau_{\mathrm{avg}}}^{k-h(k)-1} y(i)\right)=0
$$

$$
2 \zeta_{1}^{T}(k) T\left(x\left(k-\tau_{\mathrm{avg}}\right)-x\left(k-\tau_{M}\right)-\sum_{i=k-\tau_{M}}^{k-\tau_{\mathrm{avg}}-1} y(i)\right)=0
$$

$$
\tau_{M} \zeta_{1}^{T}(k) X \zeta_{1}(k)-\sum_{i=k-\tau_{m}}^{k-1} \zeta_{1}^{T}(k) X \zeta_{1}(k)
$$$$
-\sum_{i=k-h(k)}^{k-\tau_{m}-1} \zeta_{1}^{T}(k) X \zeta_{1}(k)-\sum_{i=k-\tau_{\text {avg }}}^{k-h(k)-1} \zeta_{1}^{T}(k) X \zeta_{1}(k)
$$

$$
-\sum_{i=k-\tau_{M}}^{k-\tau_{\text {avg }}-1} \zeta_{1}^{T}(k) X \zeta_{1}(k)=0
$$$$
\delta_{1} \zeta_{1}^{T}(k) Y \zeta_{1}(k)-\sum_{i=k-h(k)}^{k-\tau_{m}-1} \zeta_{1}^{T}(k) Y \zeta_{1}(k)
$$$$
-\sum_{i=k-\tau_{\mathrm{avg}}}^{k-h(k)-1} \zeta_{1}^{T}(k) Y \zeta_{1}(k)=0
$$ 
hold for any appropriate dimensions matrices $N, M, S$, and $T$, where $\zeta_{1}^{T}(k)=\left[\begin{array}{ll}x^{T}(k) & x^{T}(k-h(k))\end{array}\right]$, and semi-positivedefinite matrices

$$
X=\left[\begin{array}{ll}
X_{11} & X_{12} \\
X_{12}^{T} & X_{22}
\end{array}\right], \quad Y=\left[\begin{array}{ll}
Y_{11} & Y_{12} \\
Y_{12}^{T} & Y_{22}
\end{array}\right]
$$

Taking the forward difference of $V(k)$ and adding all the terms of (11)-(16) to $\Delta V(k)$ leads to

$$
\begin{aligned}
& \Delta V(k) \leq \zeta_{2}^{T}(k) \Phi \zeta_{2}(k)-\sum_{i=k-\tau_{m}}^{k-1} \zeta_{3}^{T}(k, i) \Theta_{1} \zeta_{3}(k, i) \\
& -\sum_{i=k-h(k)}^{k-\tau_{m}-1} \zeta_{3}^{T}(k, i) \Theta_{2} \zeta_{3}(k, i)-\sum_{i=k-\tau_{\text {avg }}}^{k-h(k)-1} \zeta_{3}^{T}(k, i) \Theta_{3} \zeta_{3}(k, i) \\
& \quad-\sum_{i=k-\tau_{M}}^{k-\tau_{\text {avg }}-1} \zeta_{3}^{T}(k, i) \Theta_{4} \zeta_{3}(k, i),
\end{aligned}
$$

where $\zeta_{2}^{T}(k)=\left[\begin{array}{llll}\zeta_{1}^{T}(k) & x^{T}\left(k-\tau_{m}\right) & x^{T}\left(k-\tau_{M}\right) & x^{T}\left(k-\tau_{\text {avg }}\right)\end{array}\right]$ and $\zeta_{3}(k, i)^{T}(k)=\left[\begin{array}{ll}\zeta_{2}^{T}(k) & y(i)^{T}\end{array}\right]$. Thus, LMIs (2) assure that $\Delta V(k)$ is negative definite.

Case II. We choose the Lyapunov function candidate

$$
V(k)=\sum_{i=1}^{5} \bar{V}_{i}(k),
$$

where the $\bar{V}_{i}(k)=V_{i}(k)$ for $i=1,2,3,4$ (are same as Case I) except for $i=5$ where

$$
\bar{V}_{5}(k)=\sum_{j=-\tau_{M}}^{-\tau_{\text {avg }}-1} \sum_{i=k+j}^{k-1} y^{T}(i) R y(i)+\sum_{j=-\tau_{M}}^{-1} \sum_{i=k+j}^{k-1} y^{T}(i) Z y(i),
$$

which yields

$$
\begin{aligned}
\Delta \bar{V}_{5}(k)= & \left(\tau_{M}-\tau_{\text {avg }}\right) y^{T}(k) R y(k)-\sum_{i=k-\tau_{M}}^{k-\tau_{\text {avg }}-1} y^{T}(i) R y(i) \\
& +\tau_{M} y^{T}(k) Z y(k)-\sum_{i=k-\tau_{M}}^{k-1} y^{T}(i) Z y(i) .
\end{aligned}
$$

Similar to (11)-(16), (11) and the following zero equations

$$
\begin{aligned}
& 2 \zeta_{1}^{T}(k) M\left(x\left(k-\tau_{m}\right)-x\left(k-\tau_{\text {avg }}\right)-\sum_{i=k-\tau_{\text {avg }}}^{k-\tau_{m}-1} y(i)\right)=0, \\
& 2 \zeta_{1}^{T}(k) S\left(x\left(k-\tau_{\text {avg }}\right)-x(k-h(k))-\sum_{i=k-h(k)}^{k-\tau_{\text {avg }}-1} y(i)\right)=0, \\
& 2 \zeta_{1}^{T}(k) T\left(x(k-h(k))-x\left(k-\tau_{M}\right)-\sum_{i=k-\tau_{M}}^{k-h(k)-1} y(i)\right)=0, \\
& \tau_{M} \zeta_{1}^{T}(k) X \zeta_{1}(k)-\sum_{i=k-\tau_{m}}^{k-1} \zeta_{1}^{T}(k) X \zeta_{1}(k) \\
&-\sum_{i=k-\tau_{\text {avg }}}^{k-\tau_{m}-1} \zeta_{1}^{T}(k) X \zeta_{1}(k)-\sum_{i=k-h(k)}^{k-\tau_{\text {avg }}-1} \zeta_{1}^{T}(k) X \zeta_{1}(k) \\
& \quad-\sum_{k-h(k)-1} \zeta_{1}^{T}(k) X \zeta_{1}(k)=0, \\
& \delta_{2} \zeta_{1}^{T}(k) Y \zeta_{1}(k)-\sum_{i=k-h(k)}^{k-\tau_{\text {avg }}-1} \zeta_{1}^{T}(k) Y \zeta_{1}(k) \\
&-\sum_{k-h(k)-1} \zeta_{1}^{T}(k) Y \zeta_{1}(k)=0 .
\end{aligned}
$$

Taking the forward difference of $V(k)$ and adding all the terms of (11) and (22) to $\Delta V(k)$ allow us to write $\Delta V(k)$ as

$$
\begin{aligned}
\Delta V(k) \leq & \zeta_{2}^{T}(k) \bar{\Phi} \zeta_{2}(k)-\sum_{i=k-\tau_{m}}^{k-1} \zeta_{3}^{T}(k, i) \bar{\Theta}_{1} \zeta_{3}(k, i) \\
& -\sum_{i=k-\tau_{\text {avg }}}^{k-\tau_{m}-1} \zeta_{3}^{T}(k, i) \bar{\Theta}_{2} \zeta_{3}(k, i) \\
& -\sum_{i=k-h(k)}^{k-\tau_{\text {avg }}-1} \zeta_{3}^{T}(k, i) \bar{\Theta}_{3} \zeta_{3}(k, i) \\
& -\sum_{i=k-\tau_{M}}^{k-h(k)-1} \zeta_{3}^{T}(k, i) \bar{\Theta}_{4} \zeta_{3}(k, i) .
\end{aligned}
$$

Thus, LMIs (4) assure that $\Delta V(k)$ is negative definite.

Remark 2. The main goal of the partitioning technique is to divide the summation interval $\left[0, \tau_{M}\right]$ of the Lyapunov function difference to as many subintervals (e.g., $\left[0, \tau_{m}\right],\left[\tau_{m}, \tau_{\text {avg }}\right]$, $\left[\tau_{\text {avg }}, h(k)\right],\left[h(k), \tau_{M}\right],\left[0, \tau_{m}\right],\left[\tau_{m}, h(k)\right],\left[h(k), \tau_{\text {avg }}\right]$, and $\left[\tau_{\text {avg }}, \tau_{M}\right]$ in the analysis) as possible. Using this technique along with the zero equations (11)-(16) and (22), we introduce free weighting matrices which increase feasibility of the resulting LMIs. 
TABLE 1: Maximum allowable upper bound $\tau_{M}$ for various $\tau_{m}$.

(a)

\begin{tabular}{|c|c|c|c|c|c|}
\hline Methods & & $\tau_{m}=6$ & $\tau_{m}=7$ & $\tau_{m}=10$ & $\tau_{m}=15$ \\
\hline Shao and Han [14] Proposition 2 & $\tau_{M}$ & 16 & 16 & 18 & 21 \\
\hline Shao and Han [14] Proposition 1 & $\tau_{M}$ & 18 & 18 & 20 & 23 \\
\hline Theorem 1 & $\tau_{M}$ & 22 & 23 & 24 & 27 \\
\hline \multicolumn{6}{|c|}{ (b) } \\
\hline Methods & & $\tau_{m}=20$ & & $\tau_{m}=25$ & $\tau_{m}=30$ \\
\hline Shao and Han [14] Proposition 2 & $\tau_{M}$ & 25 & & 30 & 34 \\
\hline Shao and Han [14] Proposition 1 & $\tau_{M}$ & 27 & & 31 & 35 \\
\hline Theorem 1 & $\tau_{M}$ & 31 & & 35 & 38 \\
\hline
\end{tabular}

\section{Numerical Simulations}

In this section, two examples are used to demonstrate the effectiveness of theorem.

Example 1. Consider the time-delay system with the following matrices:

$$
A=\left[\begin{array}{cc}
0.8 & 0 \\
0.05 & 0.9
\end{array}\right], \quad A_{h}=\left[\begin{array}{cc}
-0.1 & 0 \\
-0.2 & -0.1
\end{array}\right]
$$

Comparing the results of this work to [14], the maximum allowable upper bound (MAUB) of $\tau_{M}$ with various $\tau_{m}$ is presented in Table 1, which shows that our conditions give significantly better results.

Example 2. Consider the time-delay system with the following matrices:

$$
A=\left[\begin{array}{cc}
0.6 & 0 \\
0.35 & 0.7
\end{array}\right], \quad A_{h}=\left[\begin{array}{cc}
0.1 & 0 \\
0.2 & 0.1
\end{array}\right]
$$

This example is discussed in [15], which assumes that $\tau_{m}=2$ and $\tau_{M}=10$. For this example and by Theorem 1 in this paper, the MAUB $\tau_{M}=34$.

\section{Conclusion}

In this work, we have presented an exploitation of the partitioning oriented technique to deal with the stability problem of discrete-time systems with an interval time-varying delay. In the analysis, a new Lyapunov function was defined and some conventional inequalities were avoided resulting in relaxed stability criteria formulated into LMIs. A benchmark numerical example was given and results reflect the criteria which show significant improvement over existing results.

\section{Conflict of Interests}

The authors declare that there is no conflict of interests regarding the publication of this paper.

\section{References}

[1] E. Fridman and U. Shaked, "Stability and guaranteed cost control of uncertain discrete delay systems," International Journal of Control, vol. 78, no. 4, pp. 235-246, 2005.

[2] H. Gao and T. Chen, "New results on stability of discrete-time systems with time-varying state delay," Institute of Electrical and Electronics Engineers. Transactions on Automatic Control, vol. 52, no. 2, pp. 328-334, 2007.

[3] Y. He, Q. G. Wang, C. Lin, and M. Wu, "Delay-range-dependent stability for systems with time-varying delay," Automatica, vol. 43, no. 2, pp. 371-376, 2007.

[4] B. Zhang, S. Xu, and Y. Zou, "Improved stability criterion and its applications in delayed controller design for discrete-time systems," Automatica, vol. 44, no. 11, pp. 2963-2967, 2008.

[5] T.-S. Chiang and P. Liu, "Robust output tracking control for discrete-time nonlinear systems with time-varying delay: virtual fuzzy model LMI-based approach," Expert Systems with Applications, vol. 39, no. 9, pp. 8239-8247, 2012.

[6] J. Lin, "Exponential estimates and stabilization of discretetime singular time-delay systems subject to actuator saturation," Discrete Dynamics in Nature and Society, vol. 2012, Article ID 414373, 27 pages, 2012.

[7] Y. Wang, H. Yu, X. Zhang, and D. Li, "Stability analysis and design of time-varying nonlinear systems based on impulsive fuzzy model," Discrete Dynamics in Nature and Society, vol. 2012, Article ID 192546, 16 pages, 2012.

[8] Y. S. Moon, P. Park, W. H. Kwon, and Y. S. Lee, "Delaydependent robust stabilization of uncertain state-delayed systems," International Journal of Control, vol. 74, no. 14, pp. 14471455, 2001.

[9] P. Park, "A delay-dependent stability criterion for systems with uncertain time-invariant delays," Institute of Electrical and Electronics Engineers. Transactions on Automatic Control, vol. 44, no. 4, pp. 876-877, 1999.

[10] H. Shao, "New delay-dependent stability criteria for systems with interval delay," Automatica, vol. 45, no. 3, pp. 744-749, 2009.

[11] J. Sun, G. P. Liu, J. Chen, and D. Rees, "Improved delay-rangedependent stability criteria for linear systems with time-varying delays," Automatica, vol. 46, no. 2, pp. 466-470, 2010.

[12] M. Wu, Y. He, and H. J. She, Stability Analysis and Robust Control of Time-Delay Systems, Springer, Berlin, Germany, 2010.

[13] Y. He, G. P. Liu, and D. Rees, "Augmented Lyapunov functional for the calculation of stability interval for time-varying delay," 
IET Control Theory \& Applications, vol. 1, no. 1, pp. 381-386, 2007.

[14] H. Shao and Q.-L. Han, "New stability criteria for linear discrete-time systems with interval-like time-varying delays," Institute of Electrical and Electronics Engineers. Transactions on Automatic Control, vol. 56, no. 3, pp. 619-625, 2011.

[15] X. G. Liu, R. R. Martin, M. Wu, and M. L. Tang, "Delaydependent robust stabilisation of discrete-time systems with time-varying delay," IEE Proceedings: Control Theory and Applications, vol. 153, no. 6, pp. 689-702, 2006. 


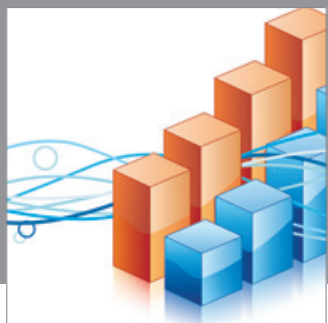

Advances in

Operations Research

mansans

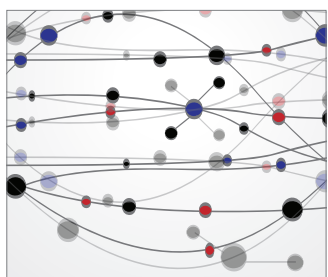

The Scientific World Journal
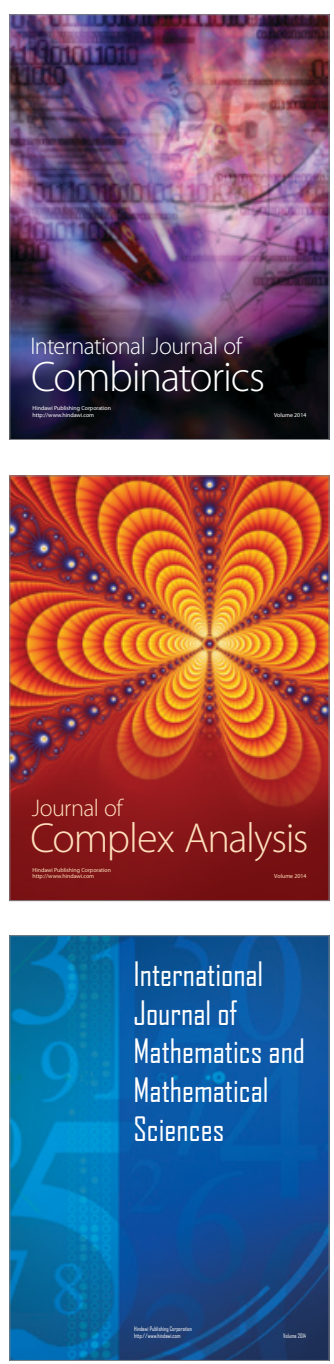
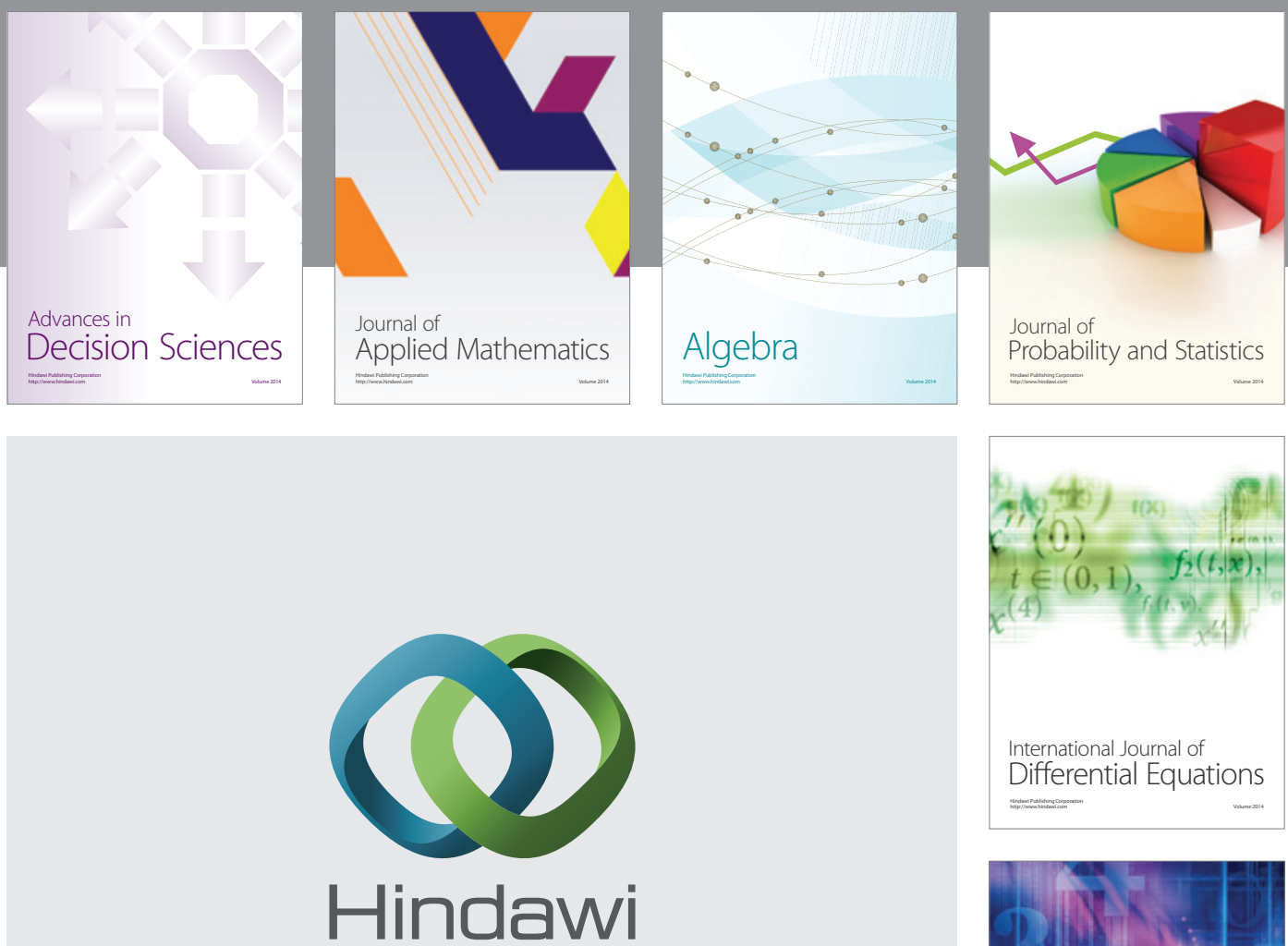

Submit your manuscripts at http://www.hindawi.com
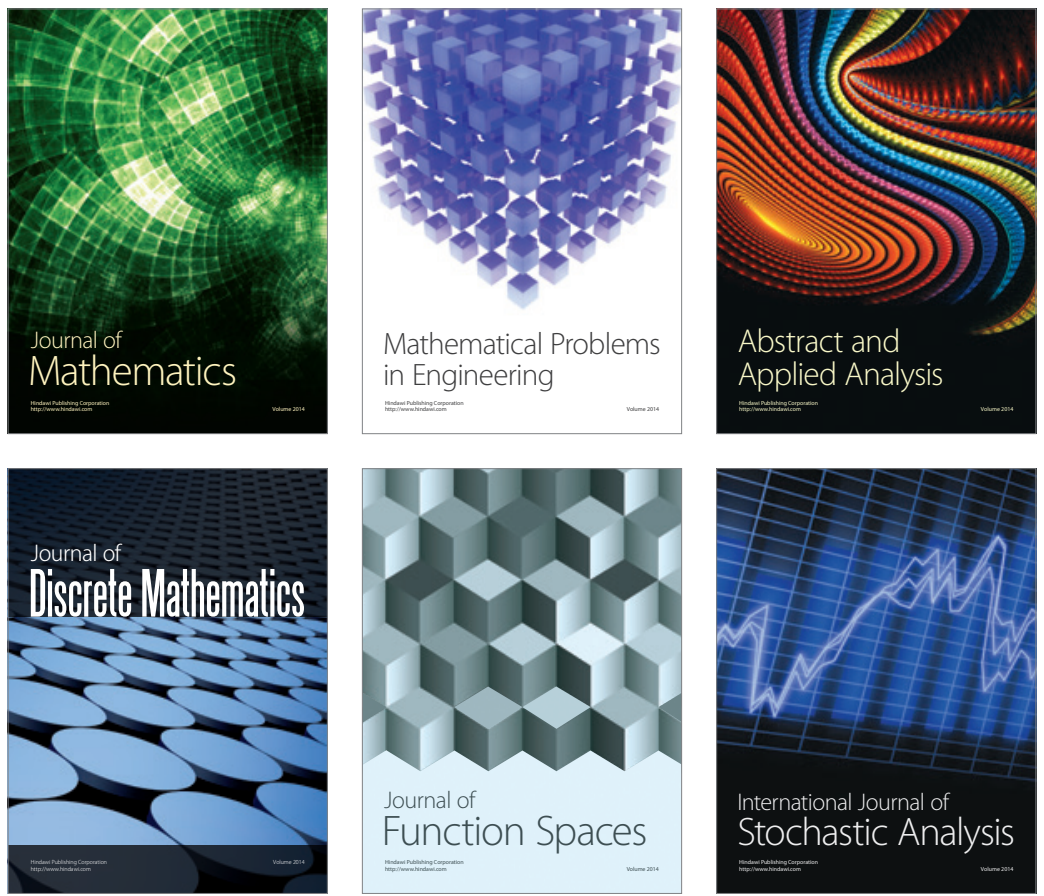

Journal of

Function Spaces

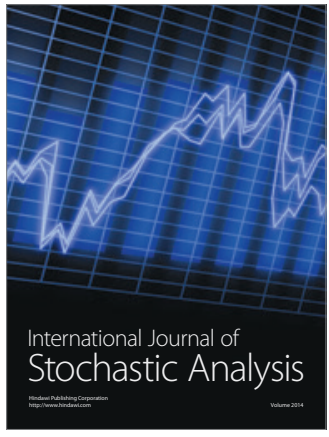

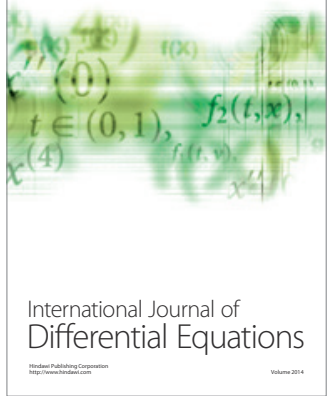
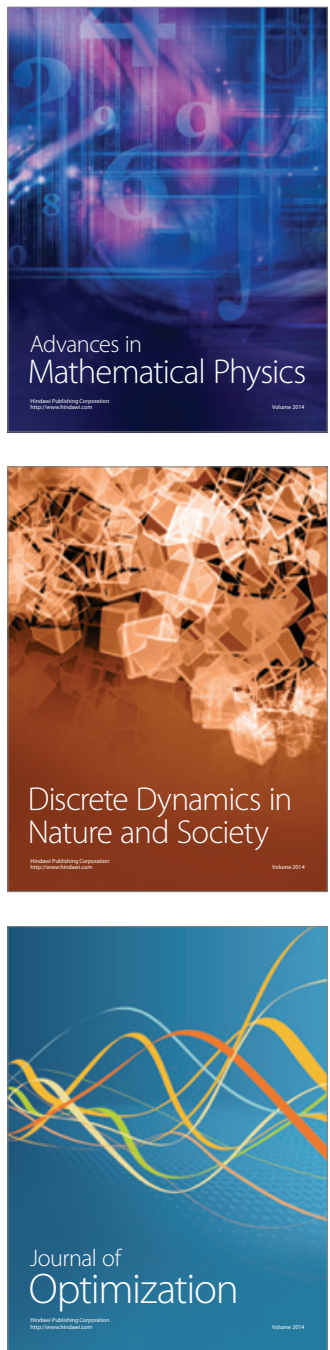